\title{
Le geste professionnel langagier de l'enseignant à l'école primaire : autorité, montée en tension et échange interrogation-réponse-évaluation
}

\author{
Christina Romain ${ }^{1}$, Véronique Rey ${ }^{2}$, et Marie Lex ${ }^{3}$

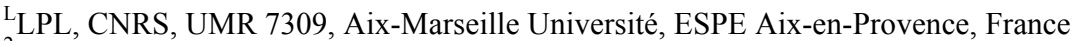 \\ ${ }^{2}$ CREDO CNRS UMR 7308, Aix-Marseille Université, ESPE Aix-en-Provence, France \\ ${ }^{3}$ Aix-Marseille Université, ESPE Aix-en-Provence, France
}

\begin{abstract}
Résumé. Dans le cadre de cet article, qui fait suite à un travail de recherche que nous avons conduit à partir du corpus recueilli par Marie Lex dans le cadre de son mémoire de fin de master métiers de l'éducation de l'enseignement et de la formation (M.E.E.F., E.S.P.E. Aix-enProvence), nous avons choisi d'analyser la relation interdiscursive et mimogestuelle entre enseignants et élèves dans trois classes de l'école Primaire afin de croiser différents indicateurs : le niveau scolaire, le geste professionnel éthique dans sa réalisation langagière et dans sa réalisation (mimogestuelle) en contexte de tension et dans le cadre de l'échange interrogation/réponse/évaluation.
\end{abstract}

\begin{abstract}
The linguistic professional gesture of the teacher in the primary school and the interactional management of the class: authority, rise in tension and exchange interrogation, response and evaluation. In the framework of this article, which follows on from a research work that we carried out based on the corpus collected by Marie Lex as part of her thesis on the end of the Master's Degrees in Education and Training (MEEF, ESPE Aix-en-Provence), we chose to analyze the interdiscursive and mimogestual relationship between teachers and pupils in three classes of the Primary school in order to cross various indicators: school level, ethical professional gesture and in its realization (mimogestual) in the context of tension and in the context of the interrogation / response / evaluation exchange.
\end{abstract}

\footnotetext{
${ }^{1}$ Corresponding author : christina.romain@univ-amu.fr
} 


\section{Cadre théorique}

\subsection{Des études antérieures à la présente étude}

Lors de nos précédentes publications, nous avons eu pour ambition de comprendre et d'analyser le fonctionnement de la relation interdiscursive en contexte de tension verbale (Romain et Rey, 2017, 2016ab, 2014 ; Rey, Romain et DeMartino, 2013, 2015 ; Romain, 2015) et de l'interroger dans sa dynamique interactionnelle de gestion de la classe en la confrontant à l'étude de la relation interdiscursive enseignant / élèves hors contexte de tension. Nous avons ainsi identifié 5 formes différenciées de montées en tension verbale en classe (Romain et Rey, 2017) :

- La montée en tension fulgurante qui repose sur des actes de langage directs sans adoucisseurs de menace, comme l'injonction.

- La montée en tension polémique à charge qui vise le discrédit d'autrui reposant sur une critique à charge explicite.

- La montée en tension contournée à visée de polémique qui repose sur des actes de langage indirects menaçants, comme l'ironie.

- La montée en tension argumentative qui vise la persuasion par la raison (argumentation reposant sur l'objet de la tension et non pas sur la personne ou un objet latéral).

- La montée en tension contournée à visée argumentative qui repose quant à elle sur un déplacement du nœud de tension. Ce point de cristallisation est caractérisé par l'émergence verbale d'un différend, reste dans ce cas dans le non-dit et est substitué dans le dit par un objet à visée de relation.

Si ces formes se différencient les unes des autres, elles sont néanmoins présentes et donc convoquées par toutes les enseignantes et tous les enseignants que nous avons pu observer. Cependant, certaines formes sont dominantes et rendent finalement compte de deux registres discursifs différenciés. Ces cinq montées en tension se répertorient donc en deux registres discursifs (Rey, Romain et DeMartino, 2013; Romain et Rey, 2014, 2016ab, 2017) :

- Le registre discursif agonal (montées en tension fulgurante, polémique, contournée à visée de polémique) (R.D. agonal).

- Le registre discursif coopératif (montées en tension argumentative, contournée à visée argumentative) (R.D. coopératif).

Ce dernier registre discursif fait écho au rôle des compétences relationnelles dans l'interaction. Des auteurs comme Blin et Deulofeu (2011) insistent sur l'importance de ces compétences qu'ils considèrent comme des outils pour prévenir et gérer les perturbations scolaires. Ils relèvent notamment l'importance de communiquer de manière non verbale (regard, posture, gestuelle) mais aussi de convoquer des compétences de communication vocales (débit, ton, rythme...). Par ailleurs, ces mêmes auteurs insistent, plus généralement, sur l'importance de coopérer avec les autres vers des objectifs communs et donc soulignent l'importance de se décentrer de son propre point de vue pour comprendre les autres et de savoir argumenter. Ainsi, dans la lignée des travaux conduits en linguistique interactionnelle de Vion (1992) (cf. le concept de règles de considération) et conformément aux travaux de Bigot (2005: 4-5), nous considérons que «le fait d'atténuer la valeur offensante ou menaçante d'un ordre constitue, une norme à respecter pour conserver le caractère non conflictuel d'une interaction [...] On rejoint ici la dialectique du sujet et du social qui se co-construisent mutuellement dans l'interaction ». Ainsi le R.D. coopératif apparait comme celui qui est le plus propice à la résolution du nœud de tension et à la poursuite de l'interaction verbale. 
Cependant, la question du choix du registre discursif pose aussi la question de la forme de l'autorité convoquée par l'enseignant en classe. Thin (2002) part du constat que les enseignants produisent un travail incessant de rappel et d'instauration de leur autorité en classe et conclut qu'il n'est pas possible de faire preuve d'autorité systématique et qu'une autorité performante conduit à faire des choix, à des compromis afin d'éviter les conflits ouverts et menaçants trop prégnants. En ce qui nous concerne, et suivant en cela les résultats des travaux de Robbes (2010), nous faisons le choix de distinguer deux formes d'autorité, l'autorité autoritariste et l'autorité éducative et de faire se superposer le registre discursif coopératif et co-énoncé à l'autorité éducative et le registre discursif agonal et surénoncé à l'autorité autoritariste (Romain et Rey, 2014). Si l'autorité autoritariste concerne « la relation où le détenteur d'une fonction statutaire exerce une domination sur l'autre afin d'obtenir de lui une obéissance inconditionnelle, sous la forme d'une soumission», l'autorité éducative, au contraire, concerne « une relation statutairement asymétrique dans laquelle l'auteur, disposant de savoirs qu'il met en action dans un contexte spécifié, manifeste la volonté d'exercer une influence sur l'autre reconnu comme sujet, en vue d'obtenir de sa part et sans recourir à la violence une reconnaissance qui fait que cette influence lui permet d'être à son tour auteur de lui-même. » (Robbes, 2010). Conformément aux travaux de Robbes, nous mettons l'accent sur un registre discursif visant notamment la négociation coopérative du moment (potentiel) de rupture interactionnelle (convoquant l'attention conjointe et orientée vers un "à venir » commun) et sur l'encouragement à produire des réponses concluantes chez les élèves par l'enseignant (enrôlement dans la tâche, étayage) dans une dimension de communication non violente visant l'épanouissement de l'élève dans son rôle et sa fonction. De même, Rivolier, Vallot, Vernusse et Médium (2011) décrivent l'autorité scolaire comme un système d'éléments " signifiants d'autorité » qui sont véhiculés principalement par la mimogestualité et qui s'organisent grâce à trois mécanismes principaux : la codification, la figuration et la ritualisation. Plus spécifiquement, Chalvin (1996) décrit les caractéristiques d'un enseignant qui fait preuve d'autorité (respect, objectifs clairs, règles énoncées peu nombreuses, droit à l'erreur, climat de sécurité et de protection) et d'un enseignant qui est autoritaire (sécheresse du ton, fait peur, communication à sens unique, règles nombreuses et imposées, rejet des contestations, tout est contraignant, punitions nombreuses). Cette description conduit Chalvin à distinguer la critique négative inhérente à l'autorité (rappel des erreurs du passé, d'une attaque personnelle ou encore d'ironie) et la critique positive inhérente à l'enseignant qui fait preuve d'autorité (information pour enclencher un changement, meilleure relation recherchée, tournée vers le futur, clarté, simplicité, franchise). Ces travaux sur l'autorité ne sont pas sans rappeler ceux de Prairat (2011) qui distingue la sanction éducative (qui vise le rappel du fonctionnement attendu, un sujet qui devient responsable et l'arrêt de quelque chose) de la punition (de laquelle découle un sentiment d'injustice).

Ainsi, dans un premier temps, la question de la relation interdiscursive entre enseignant et élèves en contexte de tension, c'est-à-dire celle du choix de la forme prise par la gestion de la tension, le registre discursif de l'enseignant et son autorité, s'est posée à nous, et nous a ensuite, dans un second temps, conduit à élargir notre champ d'étude en questionnant la relation interdiscursive hors situation de tension verbale et plus spécifiquement dans le cadre de l'échange classique interrogation, réponse, évaluation (désormais I.R.E.), que l'on retrouve dans toute interaction verbale didactique. Nous avons ainsi regardé ce qui se passait du côté des stratégies utilisées par les enseignants (les mêmes pour lesquels nous avions étudié la convocation des formes de montée en tension verbale) dans la séquence I.R.E. (Mehan, 1979 ; Sprenger-Charolles, 1983 ; Ricci, 1996 ; Romain, 2006, 2007). Nous avons ainsi pu observer que les enseignants qui convoquent un registre discursif coopératif en situation de tension verbale convoquent simultanément des stratégies diversifiées pour encourager la formulation d'une réponse concluante telles que la reformulation ou reprise 
informationnelle (ajout au minimum d'une information par le PE), la formulation interrosuspensive (amorce de réponse à compléter par l'élève), la formulation hybride (plusieurs stratégies), le concessif informationnel (mot qui n'invalide pas mais informe sur l'attente non satisfaite, apporte information de plus), ou encore le validant inductif (validation mais attente de suite à la réponse). Au contraire, les enseignants qui convoquent un registre discursif agonal en situation de tension verbale ont tendance quant à eux à convoquer des stratégies peu diversifiées (telles que la reformulation simple et la reprise simple, voir à convoquer des réfutatifs (rejet d'une réponse explicite «non») et / ou à proposer directement la réponse attendue. De même, concernant l'évaluation de clôture, si les premiers enseignants convoquent majoritairement des validations explicites, les seconds convoquent quant à eux majoritairement des validations implicites.

Ainsi, la présente étude vise d'une part à élargir ces résultats relevant de la modalité langagière à celle de la mimogestualité (elle permettra notamment de mieux comprendre la différence de registre discursif et de mimogestualité entre autorité éducative et autorité autoritariste), et d'autre part à questionner plus précisément ces résultats au regard du concept de geste professionnel. C'est pour cette raison que nous avons choisi de réunir ces différents indicateurs (gestion de la montée en tension, forme de l'autorité et stratégies d'obtention d'une réponse concluante) constitutifs de registres discursifs différenciés (R.D. coopératif et R.D. agonal) afin d'identifier la présence de ces registres chez les enseignants de notre corpus et d'observer leur lien avec la dimension mimogestuelle. Plus précisément nous allons nous demander si les mimogestes sont propres à un niveau scolaire ou bien s'ils sont propres au R.D. utilisé par l'enseignant lui-même. Notre corpus nous permettra donc de nous questionner tout à la fois sur la relation entre registre discursif, mimogestualité et niveau scolaire.

\subsection{Du geste professionnel au micro-geste professionnel}

\subsubsection{Le geste professionnel}

Nault et Fijalkow (1999) réunissent derrière la notion de gestion de classe toutes les actions produites par un enseignant qui vont avoir pour but de poser un climat de travail favorisant les apprentissages. Geste professionnel et geste de métier sont régulièrement questionnés dans cette perspective.

La notion de geste professionnel renferme toutes les actions (Sensevy, 2005) menées par l'enseignant au cours de sa séance de classe (Brudermann et Pelissier, 2008) afin de favoriser la transmission du savoir. Jorro $(2006,2007)$ distingue quant à elle le geste du métier du geste professionnel. Ainsi, si le premier est commun à toutes les enseignantes et tous les enseignants, le geste professionnel est davantage singulier en ce qu'il va par exemple réguler le premier ou encore l'ajuster en fonction des situations. Dans cette perspective, Bucheton et Soulé (2009 : 33) ont établi un modèle des gestes professionnels (voir aussi Brudermann et Pelissier, 2008) que nous allons reprendre ici : le pilotage (didactique, à travers la formulation des consignes, et d'autorité visant l'instauration de l'ordre), le tissage (forme d'étayage cherchant à donner du sens à la situation et au savoir visé et dont le but est de faciliter l'entrée dans l'activité), l'étayage (organisation de l'agir de l'enseignant accompagnant l'apprentissage: faire faire, faire verbaliser, faire comprendre ou encore transmission), l'atmosphère (climat général autorisant ou non la prise de parole d'un élève, les interactions entre élèves etc.) et le savoir (positionné au cœur de l'action de l'enseignant). Chacune d'elle regroupe des actions professionnelles que l'enseignant peut réaliser lors d'une situation d'apprentissage. Nous nous intéresserons plus particulièrement à l'atmosphère et au pilotage.

Ainsi, le présent article est l'occasion d'interroger les résultats issus de l'analyse interactionnelle des montées en tension en classe au regard de la notion de geste 
professionnel. Autrement dit, nous nous questionnons sur la constitution de nos résultats (résultats en termes de registres discursifs coopératifs favorisant la résolution de la tension et la co-énonciation en classe vs registres discursifs agonaux favorisant l'émergence récurrente de la tension et la sur-énonciation en classe) en termes de gestes professionnels langagiers interactionnels au service d'un climat favorable aux apprentissages. Nous avons privilégié le terme "langagiers » à «linguistiques » car nous adoptons un point de vue large incluant l'ensemble des régularités verbales, mimogestuelles et prosodiques (réduites à l'intensité et à la hauteur de la voix) du registre discursif convoqué par l'enseignant mais aussi tout ce qui relève de la sphère discursive (argumentation ou polémique par exemple) et pas seulement de faits linguistiques (unités lexicales et actes de langage par exemple). Si la gestion de la tension verbale tout comme l'échange I.R.E. sont constitutifs de gestes du métier d'enseignant, la forme prise par leurs différentes réalisations (choix de la forme de gestion de la tension ou encore des stratégies d'obtention d'une réponse concluante) est quant à elle constitutive de gestes professionnels, puisque chaque enseignant convoque un format de réalisation qui lui est propre. Le présent article est donc pour nous l'occasion de questionner cette construction de la relation interdiscursive, rendant compte d'une relation interpersonnelle à la fois préexistante et à la fois construite à l'occasion de l'interaction verbale en cours. C'est donc cette élaboration de la relation interdiscursive à l'occasion de l'échange que nous questionnons en tant que geste professionnel. Conformément à la classification proposée par Jorro (2006), qui présente les gestes professionnels en quatre ensembles :

- Gestes langagiers: accent didactique, langage commun, langage scolaire, langage spécifique, écoute, modalités discursives (argumentation, narration, etc.) ;

- Gestes d'ajustement de l'activité : cadrer, maintenir, accélérer, reprendre, ralentir, réguler);

- Gestes de mise en scène des savoirs : pour le professeur (aller à son bureau, vérifier une information etc.), vers l'élève-la classe, vers le savoir ;

- Gestes éthiques : formation de la communication, formation de l'appréciation scolaire (acquiescement, réprobation, jugement, interprétation, etc.).

Nous faisons entrer ce geste professionnel dans les gestes éthiques favorisant la valorisation (ou pas) de l'élève et de son travail à travers la modalité langage verbal mais aussi non verbale. Autrement dit, nous questionnons le geste éthique (manière dont l'enseignant communique avec son/ses élève(s) sur eux-mêmes ou sur leur travail) à travers son actualisation langagière, elle-même constitutive d'un geste langagier (discours de l'enseignant, convocation du registre de langue, pratique langagière), mais aussi à travers son actualisation mimogestuelle (geste, posture, mimique) et prosodique (timbre, hauteur, accentuation, etc.). Concernant ce second aspect, Duvillard (2016: 46) a proposé une observation fine des gestes professionnels en identifiant des micro-gestes qu'il définit comme «un ensemble de petits gestes sensori-moteurs et énonciatifs, conscients ou inconscients qui accompagnent et/ou portent la réalisation d'un geste ». Dans son étude, il distingue cinq micro-gestes : la posture gestuée (tenue vestimentaire, posture), la voix (timbre, intensité, débit, accentuation, articulation), le regard, l'usage du mot (notamment dans son lien avec la transmission de l'intention) et le positionnement (placement de l'enseignant dans la classe et ses déplacements).

A travers l'analyse de notre corpus, nous questionnerons donc l'articulation entre geste éthique (communication) et geste langagier (discours), mais aussi et plus largement à la macro-préoccupation de l'atmosphère (climat général) et du pilotage (formulation didactique de l'échange I.R.E.) chez l'enseignant. Notre objectif est de mieux comprendre l'enjeu interpersonnel du geste professionnel constitutif de l'élaboration de la relation interdiscursive. Mais notre objectif est aussi de questionner les micro-gestes décrits par Duvillard (2016) et de comprendre leur distribution selon la forme prise par le registre discursif des enseignants. 


\subsubsection{Le micro-geste professionnel : le rôle de la mimogestualité}

Tellier décrit le geste pédagogique comme tout « geste des bras et des mains (mais il peut aussi être composé de mimiques faciales) utilisé par l'enseignant de langue dans un but pédagogique » (2008: 41). Elle insiste sur le rôle de l'intention recherchée et elle impute trois fonctions au geste pédagogique (2006): la fonction d'information, la fonction d'animation et la fonction d'évaluation.

De même, des gestualistes posent que le geste et la parole forment un tout et s'essaient à décrire les gestes coverbaux. Ainsi, McNeill (1992) identifie quatre types de gestes coverbaux : les iconiques (gestes représentant un objet ou une action), les métaphoriques (gestes représentant un concept ou une idée), les déictiques (gestes pointant) et les battements (gestes rythmant la parole). A ces gestes sont classiquement ajoutés : les emblèmes (gestes propres à une culture) mais aussi les mimes (gestes utilisés lorsque le recours au discours est impossible).

Colletta $(2004,2005)$ prolonge la définition de ces gestes coverbaux comme étant des gestes manuels, des mimiques faciales, des changements de postures, des mouvements d'approche, de recul et de contact corporel, des évitements et des contacts oculaires. Enfin, dans le contexte de classe qui nous intéresse, Moulin (2004) a souligné que le comportement des élèves est régulièrement lié à la perception qu'ils ont du comportement de l'enseignant. Il a notamment distingué les éléments visuels des éléments sonores de la communication en classe.

Dans la lignée de ces travaux, notre postulat est que le comportement de l'enseignant agit sur la motivation et l'apprentissage des élèves. Un enseignant qui sollicite ses élèves et les encourage verra ses élèves mobiliser davantage leurs capacités intellectuelles, au contraire d'un enseignant qui a tendance à laisser ses élèves exécuter une tâche isolément. Afin d'étudier la mimogestualité par les enseignants dans les échanges étudiés, nous distinguerons :

- les éléments visuels reposant sur la proxémique (distance entre l'enseignant et l'élève), la posture, le regard et les gestes coverbaux (iconiques, déictiques, métaphoriques, battements, emblèmes)

- les éléments sonores reposant soit sur des productions vocales (ex : intensité de la voix), soit sur des productions sonores verbales buccales (ex: tut tut tut), soit encore sur des productions sonores autres (ex : taper sur la table avec la main).

\section{Méthodologie}

Notre problématique interroge la gestion différenciée de la classe en termes de gestualité de l'enseignant en fonction du niveau scolaire et en fonction des stratégies que l'enseignant convoque pour structurer la séquence I.R.E. et des formes de tension qu'il convoque dans un contexte d'autorité. Par conséquent nous proposons deux hypothèses :

- d'une part que la gestion de la séquence I.R.E. tout comme la forme prise par l'autorité de l'enseignant est différenciée suivant le niveau scolaire ;

- d'autre part que cette gestion différenciée si elle peut s'expliquer en fonction du niveau scolaire, s'explique aussi en fonction du registre discursif dominant en contexte d'autorité chez les enseignants étudiés.

Afin de vérifier nos hypothèses, nous avons comparé la gestualité de trois professeurs des écoles enseignant dans les trois cycles de l'école primaire : une première classe de 26 élèves à deux niveaux de l'école maternelle, Petite Section-Moyenne Section (PS/MS); une classe de cours élémentaire première année (CE1) de 25 élèves ; et une classe de cours moyen première année $(\mathrm{CM} 1)$ de 22 élèves. Ces trois classes sont issues de trois établissements scolaires distincts regroupant des élèves issus de milieux socioculturels différenciés. Plus précisément et pour répondre à notre problématique, nous avons filmé, 
transcrit et analysé un corpus d'une dizaine d'heures de cours afin de décrire et confronter le registre discursif en et hors tension (séquence I.R.E.) avec la mimogestualité ainsi que certaines caractéristiques prosodiques (principalement intensité et hauteur de voix) utilisées par ces trois enseignants. Nous avons commencé par découper ces transcriptions en étapes caractéristiques de la structuration du déroulement de la classe : l'entrée dans la classe/le retour dans la classe, le rituel, la présentation de la journée, l'intendance/l'appel, l'activité en classe entière (mise en activité et clôture), l'activité individuelle (mise en activité et clôture de l'activité), la mise en commun, la mise au calme de la classe, le rassemblement, la sortie en récréation, la sortie de classe. Le recensement de ces étapes nous a ensuite permis d'identifier des étapes communes aux trois niveaux scolaires qui permettaient de prendre en compte à la fois l'intégralité de la durée de la classe (en illustrant chaque étape par des extraits du corpus) mais aussi de comparer ces étapes selon le niveau scolaire.

Pour chacune de ces classes, nous avons donc étudié des étapes similaires de la journée de classe que nous avons comparé à partir de l'étude de nos indicateurs :

- les passages de rupture interactionnelle nécessitant une réparation (potentielle) de la part de l'enseignant et pour chacun d'eux nous avons relevé la forme prise par la tension et donc celle de l'autorité convoquée ;

- les stratégies utilisées (et la validation de l'échange lui-même) dans l'échange I.R.E. ;

- la mimogestualité mais aussi, dans une certaine mesure, la prosodie convoquées par les enseignants pour accompagner (ou pas) ces mêmes productions langagières.

Notre objectif étant de pouvoir décrire le registre discursif (geste langagier) de l'enseignant et l'articuler à la mimogestualité qu'il convoque en les identifiant tout deux comme un outil au service du geste éthique (communication) et plus largement de l'atmosphère de la classe et donc de l'interroger au service de la valorisation de l'élève et de son travail voire de l'enrôlement des élèves (notamment à travers les formulations didactiques dans la séquence I.R.E.) dans l'interaction didactique.

Enfin, pour conclure cette présentation méthodologique, nous tenons à préciser que nous considérons cette étude comme une étude préliminaire qui nécessitera, pour voir ses résultats confirmés ou pas, un prolongement du protocole de recherche portant sur un échantillon plus important de classes, d'établissements scolaires et donc d'enseignants et d'enseignantes.

\section{Analyse des résultats}

Dans le tableau ci-après, nous proposons un relevé synthétique d'éléments sonores (vocal, verbal buccal et sonore autre) et d'éléments visuels (gestes coverbaux, proxémique, posture et regard) relevés dans chacun des trois corpus recueillis (classes de PS/MS, de CE1 et de CM1).

Nous avons regroupé ces différents éléments suivant leur apparition dans la séquence I.R.E. et suivant leur apparition dans la séquence de tension verbale en distinguant, pour cette dernière, selon que l'enseignant adopte une autorité majoritairement autoritariste ou une autorité majoritairement éducative. Conformément à nos observations antérieures, le présent corpus rend compte chez les trois enseignants observés de la présence des deux formes d'autorité, néanmoins chacun présente une forme dominante pour une même intervention (pour le corpus analysé). C'est cette unique forme significativement dominante pour tous les extraits étudiés que nous avons fait figurée dans le tableau pour l'enseignant de CE1, tandis que nous avons fait figurer les deux formes pour les enseignantes de PS/MS et de CM1 ainsi qu'une forme supplémentaire hybride observée dans un seul extrait pour l'enseignante de PS/MS. 
Soulignons également que nous avons relevé les éléments sonores et les éléments visuels suivant qu'ils étaient conjoints à la production verbale étudiée. Ce qui explique en partie l'absence de certains indicateurs dans les extraits étudiés. Ainsi par exemple certains indicateurs sonores pouvaient être totalement absents tandis que certains éléments visuels comme la proxémique et le regard pouvaient s'avérer disjoints donc non significatifs pour la présente analyse. Concernant la dimension disjointe de ces seconds indicateurs, elle est caractéristique de l'enseignante de PS/MS et semblerait s'expliquer par une posture/proxémique et un regard sans superposition au verbal dans les extraits observés.

Une fois le tableau proposé, nous ferons une analyse pour chacune des trois classes des phénomènes observés et nous les illustrerons.

\begin{tabular}{|c|c|c|c|c|c|c|}
\hline & \multicolumn{3}{|c|}{ Eléments sonores } & \multicolumn{3}{|c|}{ Eléments visuels } \\
\hline & $\begin{array}{l}\text { Vocal } \\
\text { (intensité, } \\
\text { hauteur, } \\
\text { débit de la } \\
\text { voix, } \\
\text { enseignant } \\
\text { insiste sur } \\
\text { mot) }\end{array}$ & $\begin{array}{l}\text { Verbal } \\
\text { buccal }\end{array}$ & $\begin{array}{l}\text { Sonore } \\
\text { autre }\end{array}$ & $\begin{array}{l}\text { Gestes } \\
\text { coverbaux } \\
\text { (iconiques, } \\
\text { déictiques, } \\
\text { métaphoriques, } \\
\text { battements, } \\
\text { emblèmes) }\end{array}$ & $\begin{array}{l}\text { Posture, } \\
\text { proxémique }\end{array}$ & Regard \\
\hline \multicolumn{7}{|c|}{ P.S./M.S. } \\
\hline I.R.E. & 9 & & & $1,2,4,5,10$ & & \\
\hline A.A. & 11 & 12 & & 8,11 & & \\
\hline A.Hybride & 7 & 7 & 7 & 7 & & 7 \\
\hline A.E. & $\begin{array}{l}2,3,4,5 \\
6,9\end{array}$ & $\begin{array}{l}3,4,6, \\
9\end{array}$ & 7 & $3,4,5,9$ & & \\
\hline \multicolumn{7}{|c|}{ CE1 } \\
\hline I.R.E. & 9 & & & $2,3,5,9,12$ & 4 & 5,12 \\
\hline A.A. & $\begin{array}{l}1,2,6,7, \\
9,10,11, \\
12\end{array}$ & & 10,12 & $2,5,8,9,11,12$ & $5,6,9,11$ & 2,11 \\
\hline \multicolumn{7}{|c|}{ C.M.1 } \\
\hline I.R.E. & $\begin{array}{l}1,2,3,5 \\
6,7,12\end{array}$ & & 1,2 & $\begin{array}{l}-1,2,5 \\
\text { (+ battements et } \\
\text { gestes } \\
\text { métaphoriques) } \\
-6,12 \\
\text { (+ battements } \\
\text { ou gestes } \\
\text { métaphoriques) } \\
-3,7\end{array}$ & $2,6,7,12$ & $2,6,12$ \\
\hline A.A. & $\begin{array}{l}7,8,9,10, \\
11,13,14, \\
15\end{array}$ & & & $\begin{array}{l}-14,15(+ \\
\text { gestes } \\
\text { métaphoriques) } \\
-7,8,10\end{array}$ & $7,9,11,13$ & $\begin{array}{l}8,11, \\
15\end{array}$ \\
\hline A.E. & $2,3,6,12$ & & & $\begin{array}{l}-2,12(+ \\
\text { battements) } \\
-3,6\end{array}$ & 3,12 & $2,6,12$ \\
\hline
\end{tabular}




\subsection{La classe de PS/MS}

On observe que l'enseignante présente un R.D. que l'on peut qualifier de coopératif (les 2/3 des échanges sont concernés). En effet, si certaines situations sont propices à ce registre $(1,10)$, et d'autres (plus nombreuses) présentent ce registre dans une dimension dominante $(2,3,4,5,6,9)$. Seuls quelques extraits rendent compte d'un R.D. Agonal $(8,11,12)$. Enfin, une seule situation (7) propose un R.D. Hybride des deux formes.

\subsubsection{La séquence IRE}

On observe que ces séquences s'articulent avec de l'autorité éducative et qu'un geste (voire deux dans un seul extrait (2)) est convoqué : visuel (emblème ou déictique) ou vocal (insiste sur le mot). Voici un passage de l'extrait :

Maitresse : très bien/ alors quelle heure il est (elle regarde l'horloge) ouais on va y aller + on

va aller où $\mathrm{A}$. ?

Elève : aux toilettes

Maitresse (en parlant doucement) : tu t'appelles A. ?

A. : aux toilettes

Maitresse (en chuchotant) : Ouais::/ et après ?

A. : on se lave les mains

Maitresse : et après?

A. : on mange le pain

Maitresse (acquiesce) : et on revient pour déjeuner/ exactement

Concernant l'autorité autoritariste et l'autorité hybride, elles ne se croisent pas avec des séquences IRE (autrement dit elles ne s'articulent pas dans les extrait étudiés).

\subsubsection{La forme de l'autorité convoquée}

Il est intéressant d'observer que dans l'extrait 7 (clôture et mise en commun du travail réalisé pendant l'activité), qui est caractéristique d'un registre discursif hybride (l'enseignante oscille entre autorité autoritariste et autorité éducative), l'enseignante convoque à la fois des éléments sonores non vocaux mais aussi buccaux et vocaux auxquels s'ajoute un rôle spécifique du regard comme dans le passage suivant :

Maitresse : alors/ vous allez revenir avec moi + (tape dans les mains) on va se rasseoir là-bas/

et vous allez m'écouter + non je n'ai pas dit ça A. (baisse l'intensité de la voix)/ pas du tout/ on est en train de travailler les équilibres/ maintenant on revient s'assoir (montre du doigt le coin regroupement) + vous allez m'expliquer comment tu/ comment vous avez fait/ parce qu'il y en a qui ont réussi là (retourne vers le coin regroupement) + [...] chuuut/ S./ tu viens + bien! (Elle $\mathrm{s}$ 'assoit) +alors/ chuuut (en montrant le banc)/ assieds-toi + (en faisant non avec la main et la tête) Ah non/ attends/ tu jouais au château maintenant ton équilibre il est parti tu te débrouilles tout seul

$[\ldots]$

Maitresse : le dinosaure/ il est plus léger/ c'est pour ça que ça va vers le bas?

Elèves : non

Elève : non il est lourd

Maitresse : bah alors/ et le crayon/tu penses que c'est le contraire?

Elèves : Non!

Maitresse : si c'est vers le bas/ (en regardant l'élève qui parle, en le pointant du doigt et en faisant les gros yeux) tu te tais! (elle augmente l'intensité de sa voix) + si c'est vers le bas/ ça veut dire quoi alors ?/ Est-ce que c'est parce que/ est-ce que c'est que c'est comme ici/ que c'est lourd/ ou c'est léger?

Elève : c'est que c'est lourd

Concernant les échanges rendant compte d'autorité éducative (les plus fréquents), on observe que l'enseignante convoque dans 6 extraits sur 7 des éléments sonores vocaux 
favorable à la négociation / coopération interactionnelle (elle chuchote ou encore baisse l'intensité de sa voix comme dans le passage de l'extrait 9 ci-après afin de favoriser/d'encourager la prise de parole de A.).

Maitresse : non c'est pas lui c'est elle (geste stop de la main) + je veux pas d'histoire + regardez/ les petits ils devaient avec les balances faire des équilibres/ mettre les balances en équilibre + (montre la balance) alors là A./ qu'est-ce que tu as fait A.? (baisse l'intensité de la voix) + combien tu as mis de dinosaure ?

Elève : Deux !

(elle le regarde avec le doigt sur la bouche)

Enfin dans les trois extraits où l'enseignante convoque une autorité autoritariste, elle le fait en convoquant soit dans 1 extrait (12) un élément buccal, soit dans 1 extrait (8) deux éléments visuels (emblème et déictique), soit encore dans 1 extrait (11) une combinaison multiple d'éléments (visuels; vocaux et buccaux) dont nous rapportons un passage ciaprès :

Maitresse : chuuut/ I./ assois toi $+[\ldots]$ chuuut et je voudrais que:: alors qui est ce qui est::/ D./ est-ce que (en montrant du doigt)/ D. tu ramènes la brouette ici/ (signe de tête vas-y) prends la brouette/ (montre les caisses) tu la ramène ici/ tu ranges anneaux et petits sacs de billes $+[\ldots]$ O. ?/ O./ voilà (augmente le son de sa voix) + tu mets dans la caisse/ voilà (augmente le son de sa voix)/ c'est pas la peine de tout prendre en même temps + euuh A. (en montrant $d u$ matériel)/ tu viens m'aider à démonter ça + chhhuuut silence $+[\ldots]$ chhuuut + alors je voudrais que:: S. ? (signe doigt sur la bouche et bouche cousue)

\subsection{La classe de CE1}

On observe que l'enseignant présente principalement un R.D. Agonal $(1,3,6,7,8,9,10,11)$ et secondairement dominant $(2,4,5,12)$.

\subsubsection{La séquence IRE}

On observe que ces extraits s'articulent avec de l'autorité autoritariste et que pour accompagner sa production verbale :

- dans une moitié des extraits $(2,3,4)$ l'enseignant convoque une posture articulée à une proxémique ou un geste coverbal comme dans le passage de l'extrait 3 suivant :

Maître : de quelles phrases on a parlé déjà ?

(interroge les élèves de la tête)

Elève : <... ?>

Maître : non

Elève : $<\ldots$ ? >

Maître : de quelles phrases on a parlé la dernière fois/ qu'on a coloriées en couleurs ?

(interroge les élèves de la tête)

Elève : <... ?>

Maitre : non/ de quels types de phrase?

[...]

(interroge les élèves de la tête)

Elève : <... ?>

Maître : (il dit non de la tête) ah ben j'ai donc raison de le refaire (augmente intensité de la voix)

- dans l'autre moitié des extraits $(5,9,12)$ l'enseignant convoque un geste coverbal et un élément verbal sonore vocal / ou bien un geste coverbal et un regard comme dans le passage de l'extrait 9 suivant :

Maître (en faisant geste de la tête à C.) : C./ pourquoi t'as pas commencé ?

C. : (pas de réponse). 
Maître : on regarde au tableau (en cherchant du regard qui est l'élève qui fait du bruit) + euh $(\grave{a}$ S. en haussant les sourcils) t'arrêtes avec tes bruits ?/ Tu peux arrêter ou pas ? + Bon/ (en montrant du doigt l'addition d'O.) qu'est-ce que vous pensez de ce travail ?

Elève : elle a pas mis le égal

Maître : le égal on le met pas/ après/ autre chose

(interroge du regard)

Concernant l'autorité éducative, elle est absente des extraits étudiés.

\subsubsection{La forme de l'autorité convoquée}

Lors des échanges rendant compte d'autorité autoritariste, on observe que l'enseignant convoque dans $40 \%$ des exemples 3 à 4 gestes différenciés à la fois sonores et visuels (extraits 2, 9, 11, et 12), dans 30\% des cas 2 gestes soit visuels soit sonores soit visuel et sonore (extraits 5,6 , et 10 ), et enfin dans $30 \%$ des cas 1 geste sonore (extraits 1 , 7 , et 8 ).

Nous proposons un passage tiré de l'extrait 11 à l'occasion duquel l'enseignant enchaîne des nœuds de tension autoritariste à l'occasion desquels elle sur-énonce son point de vue sans laisser de place à une réparation du différend. Si certains extraits apparaissent comme plus nuancés que d'autres («je mettrai en cours d'acquisition» vs «tu mets ton cahier sur mon bureau »), ils sont tous constitutifs d'une intervention reposant sur un point de cristallisation du discours visant la polémique à charge (discrédit voire disqualification) de l'élève («tu mets ton cahier sur mon bureau/ je mettrai en cours d'acquisition + toi aussi/ tu es comme R./ dès que c'est un peu difficile vous laissez tomber ») :

(correction de productions d'écrits. Le maître s'est assis devant le tableau et les élèves viennent lui apporter leur cahier pour qu'ils corrigent)

Maître : euh::/ E./ S./ trouve-toi un texte! (augmente l'intensité de la voix)/ trois fois je te l'ai dit

$[\ldots]$

Maître (en croisant les bras) : J. ?/ il fallait pas te reposer toi non ?/ il fallait pas te re

(J. montre son cahier de loin)

Maître : ouais d'accord/ d'accord + et pourquoi il est retourné ton voisin ? (augmente l'intensité de la voix)

J. : euh/ parce que

Maître : toi N./ apporte-moi ton cahier de production d'écrits/ et ton cahier de brouillon + (à un élève en montrant le bureau avec la tête) euh::/ tu mets ton cahier sur mon bureau/ je mettrai en cours d'acquisition + toi aussi/ tu es comme R./ dès que c'est un peu difficile vous laissez tomber + E./ ton cahier sur mon bureau + S. ?/ les cahiers qui sont là/ mets les moi sur mon bureau s'te-plaît/ et apporte-moi ma feuille (tend la main)

$[\ldots]$

(E. parle à $N$. à côté du maître qui essaye de lire, le maître lui jette un regard réprobateur avec haussement des sourcils)

Maître (à C. en lui rendant son cahier) : Dernière chose, j'aimerais que ça soit propre. $\mathrm{Tu}$

penses que c'est faisable ?/ Allez! (augmente intensité de la voix)

$[\ldots]$

Maître (à M. en levant l'index pour le mettre en garde) : continue comme ça/ tant pis pour toi

\subsection{La classe de CM1}

On observe que l'enseignante présente un double R.D. En effet, si elle a recours à un R.D. Agonal dans plus de la moitié des interventions où elle recourt à l'autorité $(7,8,9,10,11$, $13,14,15)$, elle a recours dans tous les autres cas, y compris dans toutes les séquences I.R.E. à un R.D. Coopératif $(1,2,3,5,6,7,12)$. Il ne s'agit pas ici d'un R.D. Hybride où 
pour une même étape de la vie de la classe l'enseignante articule deux formes de R.D. mais bien de deux R.D. puisqu'ils interviennent dans des contextes d'autorité distincts en termes d'échange et que pour la séquence I.R.E. l'enseignante a exclusivement recours à un R.D. Coopératif.

\subsubsection{La séquence IRE}

Concernant les échanges faisant émerger l'autorité éducative, ils correspondent tous à cette séquence. On observe que l'enseignante, pour accompagner sa production verbale, convoque sur 7 extraits :

- dans 2 extraits $(1,2)$ des éléments sonores vocaux favorables à la négociation / coopération interactionnelle (elle baisse l'intensité ou le débit de sa voix ou augmente l'intensité pour valoriser une réponse) ainsi qu'un (geste coverbal) ou trois éléments visuels (coverbal; posture ; proxémique ; regard) et des éléments sonores ni verbaux buccaux ni vocaux. Voici un passage de l'extrait 2 :

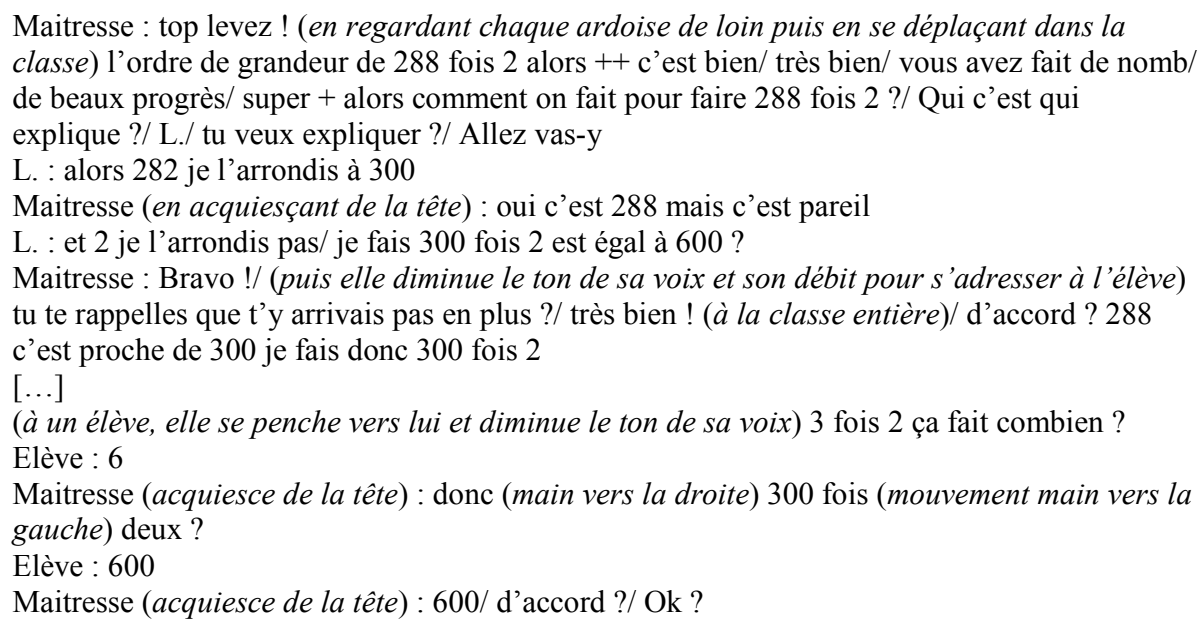

- dans 2 autres extraits $(5,3)$ des éléments sonores vocaux favorables à la négociation / coopération interactionnelle (elle baisse l'intensité ou le débit de sa voix pour réfuter une réponse ou augmente l'intensité ou le débit pour valoriser une réponse) ainsi qu'un (geste coverbal) élément visuel. Voici un passage de l'extrait 3 :

Maitresse : vous vous souvenez la semaine dernière je vous avais montré ce tableau/ on avait analysé ce tableau/ qui c'est qui se rappelle le nom du peintre ? (interroge d'un signe de tête un élève qui lève la main)

Elève : euh le tricheur de

Maitresse (elle baisse l'intensité de la voix) : alors ça c'est pas le nom du peintre/ ça c'est le TITRE du tableau

Elève : de La Tour

Maitresse (en acquiesçant de la tête) : de La Tour très bien/ et le titre du tableau comment s'appelle-t-il ? (interroge d'un signe de tête un élève qui lève la main)

Elève : le tricheur à l'as de carreau

Maitresse : oui ! très bien! le tricheur à l'as de carreau + alors on avait vu que (en pointant le tableau qu'elle tient dans une main) on avait fait un partage de cartes à partir de là vous vous rappelez?

- dans 1 autre extrait (7) des éléments sonores vocaux principalement favorables à la négociation / coopération interactionnelle (elle baisse l'intensité ou le débit de sa voix pour 
réprimander ou questionner et inversement augmente l'intensité ou le débit de sa voix pour valoriser un ou des élèves) ainsi que deux éléments visuels (coverbal; posture; proxémique). Voici un passage de l'extrait 7 :

Elève : 12

Maitresse : 12 !/ très bien bravo + chacun en aura 12 (en pointant « 24 » au tableau) la moitié de 24 c'est 12 + d'accord ?/ si tu partages en (place sa main au milieu sur la fiche accroché au tableau pour séparer en deux les biscuits) deux/ la moitié c'est 12/ ok?

(l'élève va s'assoir)

Maitresse: très bien/ (en pointant du doigt l'opération au tableau) qui avait écrit comme ça ?/

Qui a fait juste?

(les élèves lèvent la main)

Maitresse : je vous félicite !/ très bien/ bravo/ impeccable

- enfin, dans seulement 2 extraits $(6,12)$ des éléments sonores vocaux non favorables à la négociation / coopération interactionnelle (elle augmente l'intensité ou le débit de sa voix ; insiste sur un mot) ainsi que trois éléments visuels (coverbal; posture; proxémique; regard). Voici un passage de l'extrait 12 :

Maitresse (en réfutant d'un signe de tête et regard réprobateur) : quel déterminant ? / non/ le complément du nom/ il commence par quoi ?

Elève : (ne répond pas)

Maitresse (en allant vers lui) : montre-moi où est le déterminant/ (il lui montre sur son cahier) c'est pas un déterminant ça/ c'est une préposition d'accord ?/ Ça s'appelle une préposition/ les déterminants ils sont là/ (pointe du doigt sur son cahier) devant le nom un une le la/ d'accord ?

\subsubsection{La forme de l'autorité convoquée}

On observe que les $2 / 3$ des extraits $(7,8,9,10,11,13,14,15)$ contiennent de l'autorité autoritariste où la production verbale de l'enseignante est accompagnée :

- d'éléments sonores vocaux (insiste sur des mots; augmente l'intensité ou du débit de la voix)

- et par des éléments visuels : pour la moitié d'un geste visuel coverbal et pour l'autre moitié de deux gestes visuels (coverbal et regard; coverbal, posture et proxémique; posture, proxémique et regard).

Voici un passage de l'extrait 11 :

Maitresse (se tourne vers les élèves) : vous avez fini les enfants?

Elèves : non

Maitresse : ne parlez pas alors/ d'accord (insiste sur le mot)?

Elèves : oui

(L'enseignante parcourt la classe du regard)

Maitresse : concentrez-vous

Ensuite, $1 / 3$ des extraits $(2,3,6,12)$ contient de l'autorité éducative où la production verbale de l'enseignante est accompagnée :

- d'éléments sonores vocal (diminution de l'intensité ou du débit de la voix ; chuchotement) - et par des éléments visuels qui sont tous au nombre de deux (coverbal et regard ; coverbal, posture et proxémique)

- mais aussi dans un cas au nombre de trois (coverbal, regard, posture et proxémique).

Voici un passage de l'extrait 2:

Maitresse : levez (regarde les ardoises) + (en baissant l'intensité de la voix et en direction de L.)

je vois pas L./ si tu tournes pas vers moi + d'accord + N./ (baisse intensité de la voix) quand tu 
lèves l'ardoise c'est (lève les points pour montrer comment tenir l'ardoise) comme ça si tu fais (main levé paume vers le ciel) ça face au plafond/ je ne vois pas/ tu comprends ? (acquiesce de la tête)

\section{Synthèse}

\subsection{Vers une confirmation de l'hypothèse de différences selon les niveaux scolaires}

Premièrement, on observe que concernant la séquence IRE si les trois enseignants (dans les extraits étudiés en tout cas) convoquent des éléments sonores vocaux et des éléments visuels coverbaux accompagnant majoritairement leurs productions verbales, les enseignants de CE1 et de CM1 ont également recours à des gestes visuels (posture, proxémique et regard pour l'enseignant de CE1) et aux trois formes de gestes visuels (coverbaux, posture, proxémique et regard pour l'enseignante de CM1) mais aussi à des éléments sonores vocaux visant majoritairement une diminution de l'intensité et/ou du débit (alors que dans ce dernier cas, les deux autres enseignants insistent sur le/les mots toujours pour l'enseignante de CM1).

Deuxièmement, concernant les échanges d'autorité, on observe que l'enseignant de CE1 (présentant un R.D. favorisant un recours dominant à l'autorité autoritariste), l'enseignante de CM1 (présentant un R.D. favorisant tant un recours à l'autorité éducative qu'à l'autorité autoritariste), tout comme l'enseignante de P.S./M.S. (présentant un R.D. favorisant un recours dominant à l'autorité éducative) accompagnent leurs productions verbales d'éléments visuels coverbaux mais avec, pour chaque enseignant, des caractéristiques spécifiques :

- L'enseignant de CE1 les accompagne d'éléments à la fois d'éléments sonores vocaux (augmentation de l'intensité ou du débit de sa parole) et non vocaux (tape sur le bureau par exemple) mais aussi d'éléments visuels comme la posture et la proxémique ainsi que le recours au regard.

- L'enseignante de P.S./M.S. accompagne les éléments visuels coverbaux d'éléments essentiellement sonores mais différents de ceux de l'enseignant de CE1: il s'agit d'éléments sonores vocaux (diminution de l'intensité ou du débit de sa parole).

- Enfin, concernant le cas de l'enseignante de CM1, il est intéressant de relever qu'elle emprunte les caractéristiques des deux autres enseignants. En effet, les points communs se recoupent en fonction de la forme d'autorité convoquée... La seule différence concerne les éléments visuels qui sont plus diversifié dans le cadre de l'autorité éducative en CM1 par rapport à la PS/MS. Elle accompagne les éléments visuels coverbaux des autres éléments visuels (autorité autoritariste : posture, proxémique ou regard ; autorité éducative : posture, proxémique et/ou regard) et d'éléments sonores exclusivement vocaux mais avec une différence en fonction de la forme d'autorité (autorité éducative : diminution de l'intensité ou du débit / débit voire chuchotement ; autorité autoritariste : augmentation de l'intensité / débit ; insiste sur un mot).

Il est intéressant de relever que l'enseignante de CM1 a recours à davantage de gestes sonores et visuels tant en autorité autoritariste (comme l'enseignant de CE1) qu'en autorité éducative (ce qui est différent de l'enseignante de PS/MS). 


\subsection{Vers une confirmation de l'hypothèse de différences selon les registres discursifs (autoritariste vs éducatif) des enseignants}

On peut donc observer que les enseignants de PS/MS et de CE1 convoquent des éléments sonores et visuels globalement différenciés et qu'ils sont davantage propices à la négociation pour l'enseignante de $\mathrm{PS} / \mathrm{MS}$ et davantage propice à la tension pour l'enseignant de CE1.

Quant à l'enseignante de CM1, on peut observer qu'elle convoque un nombre d'éléments sonores et visuels supérieurs aux deux autres enseignants et que dans le même temps elle convoque suivant les étapes de l'autorité éducative ou de l'autorité autoritariste.

Plus précisément,

- on observe des différences selon la forme prise par l'autorité : ainsi l'autorité autoritariste convoque systématiquement des éléments sonores accentuant l'intensité et/ou le débit de parole de l'enseignant, le fait d'insister sur un mot également (cf. enseignants de CM1 et de CE1).

- on observe ces mêmes enseignants, qui utilisent l'autorité autoritariste, ont également recours à des procédés de constitution de la séquence IRE qui ne favorisent pas la coopération avec les élèves, la valorisation des réponses, etc.

On observe encore que les enseignantes de PS/MS et de CM1 convoquent tous les deux l'autorité éducative en contexte de tension et de séquence IRE ce qui favorise la coopération et la relation interdiscursive au service de la formulation de réponse concluante (et ce indépendamment donc du niveau scolaire). Au contraire, l'enseignant de CE1 articule IRE et autorité autoritariste (en et hors IRE) et ne favorise donc pas la formulation de réponse concluante.

Il s'avère bien entendu que ces enseignants ont des classes différentes qui peuvent potentiellement expliquer aussi ce phénomène. Néanmoins, du fait de leur recours à des autorités différenciées ou pas (autoritariste vs éducative), il serait intéressant de poursuivre ce travail en croisant les profils (par exemple en trouvant une classe de cel avec un-e enseignant-e convoquant une autorité majoritairement éducative et une classe de P.S./M.S. avec un-e enseignant-e convoquant une autorité majoritairement autoritariste).

On observe enfin que l'enseignant de CE1 et, encore plus, l'enseignante de CM1 utilisent des gestes plus variés que l'enseignante de PS/MS. On observe qu'en situation d'autorité les gestes sont plus variés pour toutes les enseignantes et tous les enseignants mais davantage encore pour ceux qui recourent à l'autorité autoritariste.

A ce stade de notre présente recherche, nous avons donc commencé à répondre favorablement à nos deux hypothèses de départ. Cependant, nous tenons à modaliser nos résultats du fait même de la limite de l'échantillon constitutif de notre corpus (et de sa représentativité). Comme nous l'avons déjà souligné, nos résultats méritent maintenant d'être confronté à l'analyse d'un corpus plus important de classes et donc d'enseignants.

\section{Conclusion}

Les résultats de notre étude permettent néanmoins de conclure que des différences existent entre les trois niveaux scolaires :

- Séquence I.R.E. : si les trois enseignants accompagnent leurs productions verbales d'éléments de type visuel coverbal, l'enseignante de CM1 produit des formes complémentaires (métaphoriques et battements), les enseignants de CE1 et de CM1 convoquent en plus des éléments visuels coverbaux des éléments de l'ordre de la posture, de la proxémique et du regard, et l'enseignante de CM1 convoque quant à elle des éléments sonores ni vocaux ni verbaux buccaux. 
- La forme dominante d'autorité : si l'enseignante de PS/MS a recours à l'autorité éducative, l'enseignant de CE1 a recours à l'autorité autoritariste et l'enseignante de CM1 convoque tantôt l'une et tantôt l'autre en contexte de tension et exclusivement l'autorité éducative pour la séquence I.R.E.

Cependant, concernant les gestes sonores et visuels accompagnant ces autorités, la différence selon les niveaux n'est plus opérante. En effet, les enseignants convoquant l'autorité éducative utilisent de façon spécifique les gestes sonores (diminution de l'intensité et / ou du débit) par rapport à ceux convoquant l'autorité autoritariste (augmentation de l'intensité et / ou du débit), de même ils utilisent les éléments visuels de façon différenciée (l'autorité autoritariste convoque davantage de gestes visuels variés).

Les différences observées ne sont donc pas exclusivement corrélées au niveau scolaire mais aussi à la forme prise par le R.D. de l'enseignant. Au surplus, nous avons également observé que concernant la constitution de la séquence I.R.E. les stratégies convoquées par les enseignants laissant une place significative à l'autorité autoritariste ne sont pas favorable à la négociation visant la formulation d'une réponse concluante.

Il s'avère que le R.D. Coopératif favorise la négociation en termes de réparation $\mathrm{du}$ nœud de rupture interactionnelle (potentielle) en réorientant l'attention conjointe sur un « à venir » à partager en collaboration. Au contraire, le R.D. Agonal ne favorise pas la négociation et consiste pour l'enseignant à imposer son point de vue à l'élève.

Notre étude a ainsi permis de mettre en avant des outils favorisant le R.D. Coopératif et pas seulement en termes de résolution de conflit mais aussi en termes de structuration de la séquence I.R.E. donc en contexte généralement hors tension : l'autorité éducative et les stratégies (mises en mots par les enseignants) de recherche de formulation de réponse concluante (chez l'élève).

Finalement, notre étude aura permis de mettre en lumière l'existence de deux ensembles de gestes d'autorité, se retrouvant chacun tant à l'occasion de la gestion des montées en tension verbale que lors de la structuration des activités de la classe (introduction, clôture, transition) : le premier correspondant à des gestes d'autorité éducative caractérisant le R.D. Coopératif et le second correspondant à des gestes d'autorité autoritariste caractérisant le R.D. Agonal. Ces résultats permettent d'identifier l'articulation avec le verbal des gestes coverbaux, des gestes sonores, mais aussi relevant de la proxémique, de la posture et du regard, en croisant l'impact à la fois sur la structuration des activités mais aussi sur la gestion des moments de tension vebrale afin d'améliorer ou à tout le moins de mieux comprendre les enjeux de la gestion interactionnelle de la classe.

\section{Références}

Bigot, V. (2005). Quelques questions de méthodes pour une recherche sur la construction de la relation interpersonnelle en classe de langue. Primauté des données et construction de savoirs. Le Français dans le monde. Recherches et applications, Paris : Français dans le monde, pp.42-53.

Blin, J.-F. \& Deulofeu, C.-G. (2011). Classes difficiles. Des outils pour prévenir et gérer les perturbations scolaires. Delegrave Pédagogie et Formation : Lassay-les-Châteaux

Brudermann, C. et Pelissier, C. (2008). Les gestes professionnels de l'enseignant : une analyse pédagogique et une représentation informatisée pour la formation - L'exemple des langues étrangères. Revue internationale des technologies en pédagogie universitaire, $5(2), 21-33$.

Bucheton, D. et Soulé, I. (2009). « Les gestes professionnels et le jeu des postures de l'enseignant dans la classe : un multi-agenda de préoccupations enchâssées ", Éducation et didactique [En ligne], vol $3-\mathrm{n}^{\circ} 3 \mid$ Octobre 2009, mis en ligne le 01 octobre 2011, 
consulté le 30 septembre 2016. URL : http://educationdidactique.revues.org/543 ; DOI : 10.4000/educationdidactique. 543

Chalvin, M.-J. (1996). Prévenir Conflits et Violence. Baume-les-Dames : Nathan Pédagogie.

Colletta, J. M. (2004). Le développement de la parole chez l'enfant âgé de 6 à

11 ans : corps, langage et cognition. Sprimont : Mardaga.

Colletta, J. M. (2005). Communication non verbale et parole multimodale : quelles implications didactiques? Le Français dans le Monde, Recherches et Applications, (39), 32-41.

Duvillard, J. (2016). Ces gestes qui parlent : l'analyse de la pratique enseignante. Paris : ESF.

Jorro, A. (2006). L'agir professionnel de l'enseignant. Conférence au séminaire de la Recherche du Centre de Recherche sur la Formation, Paris.

https://halshs.archives-ouvertes.fr/halshs- 00195900/document.

Jorro, A. (2007). L'agir professionnel de l'enseignant. Conférence au séminaire de Recherche $d u$ Centre de Recherche sur la Formation - 28 février 2006 - CNAM, Paris. Archives ouvertes, version 1 du 11 décembre 2007.

Mehan, H. (1979). Learning Lessons. Social Organization in the classroom. Cambridge, Massachusetts, \& London, England : Harvard University Press.

Mcneill, D. (1992). Hands and mind: what gestures reveal about thought. Chicago : University of Chicago Press.

Moulin, J. F. (2004). Le discours silencieux du corps enseignant. La communication non verbale du maître dans les pratiques de classe. Carrefours de l'éducation, (17), 142-159.

Nault, T. et Fijalkow, J. (1999). Introduction. La gestion de la classe : d'hier à demain. Revue des sciences de l'éducation, 25(3), 451-466.

Prairat, E. (2011). La sanction en éducation. Paris : P.U.F.

Rivolier, A., Vallot, F. \& Vernusse, P. (2011). L'autorité à l'école. Médium, 2(27), p120-139.

Robbes, B. (2010). L'autorité éducative dans la classe. Douze situations pour apprendre à l'exercer. Paris : ESF.

Romain, C. (2015). Interactions langagières entre enseignant et élèves au collège (milieu facile vs milieu difficile) : vers un contenu différencié en fonction de l'appartenance socioculturelle des élèves ou en fonction du « registre discursif » des enseignants?. In Auger, N. \& Romain C. Violence verbale et école. Coll. Enfance et langages. L'Harmattan, 49-82.

Romain, C. (2007). «Vers un contenu sociodifférencié de l'échange interrogatifinformationnel ? ». Dans Giacomi et E. Vargas (éds.). Pratiques sociales et didactique des langues (75-97). Aix-en-Provence : Presses Universitaires de Provence.

Romain, C. (2006). Stratégies d'obtention d'une formulation concluante en séquence ternaire et séquence latérale. Cahiers de la recherche et du développement -Skholê, Hors Série 1, 91-104.

Romain, C. et Rey, V. (2017). Les normes linguistiques de la gestion de la tension chez l'enseignant 
dans l'espace de la classe en école primaire. In L. Gaudin-Bordes \& M. Monte, Normes textuelles et discursives : émergence, variations et conflits "Annales littéraires de l'université de Franche-Comté". Besançon : Presses Universitaires de Franche-Comté.

Romain, C et Rey, V. (2016a). Stratégies d'affirmation et gestion (co)-énonciative de la tension verbale dans l'interaction didactique. Testi e linguaggi, Rivista di studi letterari, linguistici e filologici dell'Universita di Salerno. Vol. 10. p.143-162.

Romain, C. \& Rey, V. (2016b). Enjeux sémantiques des actes de langage menaçants en classe : de la menace argumentative positive à la menace polémique négative. In Olga Galatanu, Ana-Maria Cozma et Abdelhadi Bellachhab (dir.), Représentations du sens linguistique : les interfaces de la complexité, (pp. 321-336). Bruxelles : Peter Lang.

Romain, C. \& Rey, V. (2014). Montée en tension, répétition lexicale, co-énonciation et surénonciation dans l'interaction entre enseignant et élève(s). In Franck Neveu, Peter Blumenthal, Linda Hriba, Annette Gerstenberg, Judith Meinschaefer et Sophie Prévost (Eds.), Actes du Congrès Mondial de Linguistique Française - CMLF 2014, Université libre de Berlin, 2014, Institut de Linguistique Française, Didactique et enseignement, français langue maternelle, français langue seconde. EDP Sciences http://www.shsconferences.org/articles/shsconf/pdf/2014/05/shsconf_cmlf14_01062.pdf p. 2165-2178

Rey, C., Romain, C. \& DeMartino, S. (2015). Espaces de tensions interactionnelles à l'école primaire (milieu scolaire traditionnel et milieu scolaire hospitalier) : relation interpersonnelle, gabarits de langue, compétences langagières et effet miroir. In Auger, N. \& Romain C. Violence verbale et école. Coll. Enfance et langages. L'Harmattan, 83-119.

Rey, V., Romain, C. \& DeMartino, S. (2013). Tensions interactionnelles en milieu de scolarisation hospitalière et scolaire: de la détresse langagière aux pratiques langagières relationnelles et médiatrices favorisant des espaces sociaux apaisés. In Gradoux Xavier, Jeanneret Thérèse \& Anne-Christel Zeiter (Eds) (2014). "Rôle des pratiques langagières dans la constitution des espaces sociaux pluriels d'aujourd'hui. Actes du colloque VALSASLA 2012 (Lausanne, 1-3 février 2012)". Bulletin suisse de linguistique appliquée, Numéro spécial hiver 2013. Neuchâtel: Centre de linguistique appliquée, p.151-169 http://doc.rero.ch/record/11876

Ricci, L. (1996). Le dialogue interrogatif ou les deux faces d'une liberté didactique. Les carnets du Cediscor, 4, 131-151.

Sensevy, G. (2005). Lettre de l'Association Internationale pour la Recherche en Didactique du Français, 36, 1, 4-6.

Sprenger-Charolles, L. (1983). « Analyse d'un dialogue didactique : l'explication du texte », Pratiques, 40.

Tellier, M. (2006). L'impact du geste pédagogique sur l'enseignement/l'apprentissage des langues étrangères : Etude sur des enfants de 5ans (Thèse de doctorat non publiée, Université Paris 7-Denis). Repéré à https://tel.archives-ouvertes.fr/tel-00371041.

Tellier, M. (2008). Dire avec des gestes. Le Français dans le Monde, Recherches et applications, (44), 40-50.

Thin, D. (2002). Le cas des collèges de quartiers populaires. Revue Française de Pédagogie, $\mathrm{n}^{\circ} 139$, 21-30.

Vion, R. (1992), La communication verbale. Analyse des interactions, Paris, Hachette.

Vygotsky, L.S. (1985). Pensée et langage, (1934), Éd. française Messidor. 